\title{
Os usos flexíveis do conceito de variável na Educação Básica: Um estudo envolvendo as Equações Diofantinas Lineares
}

\author{
The flexible usages of the variable concept at basic education: \\ A study involving Linear Diophantine Equation
}

\author{
Wagner Marcelo Pommer \\ Universidade Federal de São Paulo, SP, Brasil \\ wmpommer@gmail.com
}

\begin{abstract}
Resumo
Em diversos documentos curriculares para a Educação Básica o conceito de variável é brevemente citado, veiculado como uma noção paramatemática, intrínseca ao desenvolvimento das funções, sem maiores referências. Este artigo tem por objetivo apresentar e discutir o contexto das Equações Diofantinas Lineares como possível tema para explorar o conceito de variável, de forma flexível, na Educação Algébrica. O referencial teórico se encontra em Küchemann (1981), Usiskin (1995) e Ursini e Trigueros (2001), pesquisadores que consideram que as variáveis podem assumir distintos papéis: incógnita, número generalizado e em relacionamento funcional. Nesses moldes, a compreensão do conceito de variável perpassa algumas potencialidades. Nos inspiramos na Análise de Conteúdos, conforme Bardin (2004), como referencial metodológico para a busca de contextos que favorecessem tal quadro. Na preanálise realizada consideramos a epistemologia envolvendo o tema das Equações Diofantinas Lineares como possível caminho para a exploração dos usos flexíveis do conceito de variável. A análise desse tema revelou que as Equações Diofantinas Lineares perpassam a aquisição das seguintes potencialidades, expressas em Ursini e Trigueiros (2001): execução de cálculos e operações simples com os símbolos literais; oportunizam um contexto de integração dos usos flexíveis da variável; permitem situar as vantagens dos diferentes usos do conceito de variável.
\end{abstract}

Palavras-chave: Variável. Equação Diofantina Linear. Álgebra. Educação Básica

\begin{abstract}
In many curricular documents for Basic Education the variable concept is briefly quoted, seen as a paramathematics notion, intrinsic to the development of functions, without further references. This article aims to present and discuss the context of Linear Diophantine Equation as a possible theme to explore the flexible uses of the variable concept in algebraic education. The theoretical considerations is based upon Küchemann (1981), Usiskin (1995) and Ursini;Trigueros (2001), researchers who believe that variables can assume different roles: unknown, generalized number and in a functional relationship. In that scenary, the understanding of the variable concept pervades some potential ways. We were inspired at Content Analysis, described in Bardin (2004), as metodological referencial to search contexts that favour such framework. In preanalisys realized we consider the epistemology present on Diophantine Linear Equations theme as a possible way to explore the flexible uses of the variable concept. The analysis revealed that the Linear Diophantine Equations allow the acquisition of the following potentialities, expressed in Ursini and Trigueiros (2001): executing calculations and simple operations with the literal symbols; create a context of integration to the variables flexible uses; allow situating some advantages on the different uses of the variable concept.
\end{abstract}

Keywords: Variable. Linear Diophantine Equation. Algebra. Basic Education 


\section{Introdução}

A Álgebra é uma importante área do conhecimento matemático, conforme se observa no programa do ensino básico, descrito em Brasil (1997, 1998, 2000). A Álgebra está inserida dentro de diversos ramos da matemática do Ensino Médio, particularmente no estudo das equações polinomiais e das funções, sendo utilizada como ferramenta para o estudo do “[...] comportamento de certos fenômenos tanto do cotidiano, como de outras áreas do conhecimento, como a Física, Geografia ou Economia" (BRASIL, 1998, p.45).

Assumimos, então, a função da Álgebra como instrumento fundamental na gênese do pensamento matemático e científico, assim como presente na intenção de formação para a cidadania e trabalho. Em contrapartida, o ensino:

[...] da Álgebra vem apresentando tantos fracassos que passou a ser também um elemento de exclusão social, uma vez que, os que não conseguem aprendê-la, vêem formar-se diante de si barreiras intransponíveis para a ascensão do conhecimento (CASTRO, 2005, p.2).

\section{0 conceito de variável no material curricular brasileiro}

Um primeiro mapeamento sobre o tema das variáveis pode ser realizado no modo como é apresentado e tratado nos documentos oficiais de Ensino Fundamental e Médio.

Uma primeira aproximação dos PCN, Brasil (1997) com relação às variáveis relata:

[...] que nas séries finais do Ensino Fundamental os trabalhos algébricos deverão ser ampliados; trabalhando com situaçõesproblema, o aluno reconhecerá diferentes funções da Álgebra (como modelar, resolver problemas aritmeticamente insolúveis, demonstrar), representando problemas por meio de equações (identificando parâmetros, variáveis e relações e tomando contato com fórmulas, equações, variáveis e incógnitas) e conhecendo a 'sintaxe' (regras para resolução) de uma equação (p. 39).

Na descrição dos PCNEM, Brasil (2000), um dos objetivos da área de Ciências da Natureza, Matemática e suas Tecnologias é a constituição de competências e habilidades que permitam ao educando identificar:

[...] variáveis relevantes e selecionar os procedimentos necessários para produção, análise e interpretação de resultados de processos ou experimentos científicos e tecnológicos; identificar, analisar e aplicar conhecimentos sobre valores de variáveis, representados em gráficos, diagramas ou expressões algébricas, realizando previsão de tendências, extrapolações e interpolações, e interpretações (p. 95-96).

Nas Orientações Educacionais Complementares aos Parâmetros Curriculares Nacionais (PCN+), Brasil (2005), na área de Matemática retomam-se as competências delineadas acima: "Identificar em dada situaçãoproblema as informações ou variáveis relevantes e elaborar possíveis estratégias para resolvê-la" (p. 112). No mesmo documento, tais descrições são retificadas em relação às demais áreas, de modo similar.

Em Brasil (1998) e em Brasil (2000) há referência ao trabalho com a Álgebra no Ensino Fundamental e Médio em quatro dimensões: 'Aritmética Generalizada', do ponto de vista 'Funcional', explorando o tema das 'Equações' e, por último, no enfoque 'Estrutural', conforme se descreve no quadro 01.

Quadro 01: A perspectiva dos PCN, no Ensino Fundamental II (BRASIL, 1998, p. 116).

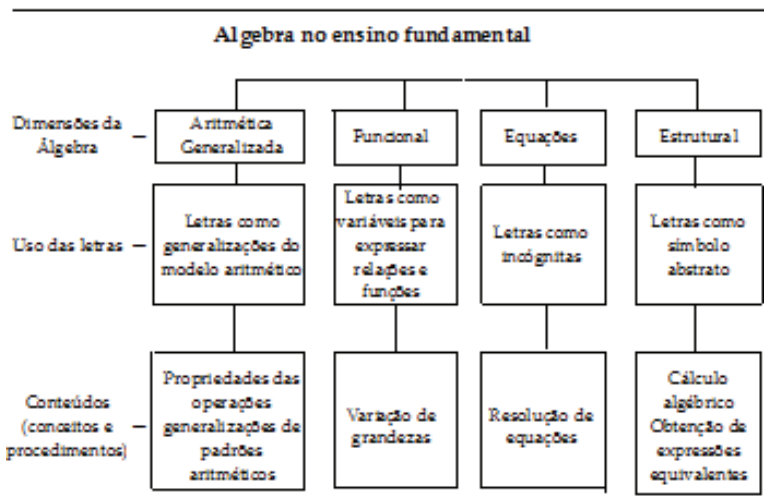

Uma análise inicial do Quadro 01 revela quatro caminhos, explicitamente distintos, para o trabalho com as letras no ensino básico. Porém, o mapa não indica e nem explora as possíveis conexões e interelações existentes entre as quatro grandes dimensões: 'Aritmética Generalizada', 'Funcional', 'Equações' e 'Estrutural'. 
Porém, é justamente “[...] na passagem da Álgebra Aritmética para a Álgebra das Equações e desta para a Álgebra Funcional [onde] há uma ressignificação de conceitos" (SILVA; ANDRADE, 2012, p.2).

Outro ponto a se considerar nas propostas curriculares é que esta situa a noção de variável associada exclusivamente como a letra que expressa relações no quadro das funções.

Em relação a este aspecto, Schoenfeld e Arcavi (1988) fazem uma observação: nos materiais curriculares existe uma suposição implícita que o conceito da variabilidade é possível de ser aprendido pela exploração de situações pragmáticas, ao se abordar o tema das funções, sem a intenção de uma discussão conceitual para se desenvolver a noção de variável.

Outra observação pode ser feita levando-se em consideração a argumentação: “O estudo das grandezas variáveis deu origem ao conceito de função" (BRASIL, 1998, p. 24). Porém, não encontramos neste material um desenvolvimento em relação a esta afirmação.

A Proposta Curricular do Estado de São Paulo, São Paulo (2008) indica um caminho para o trabalho com o tópico Funções: "Noções básicas sobre função; A ideia de variação; Construção de tabelas e gráficos para representar funções de 1o e 2o graus" (p. 53). Porém, tal documento não indica modos de se desenvolver o percurso proposto.

Com base nestes relatos, podemos considerar que o conceito de variável se aproxima de uma noção paramatemática. Segundo Chevallard; Bosch; Gascón (2001), a noção paramatemática representa objetos de saber auxiliares, necessários ao ensino de objetos matemáticos (no caso, o tema das funções). Tais noções não se constituem em objeto de ensino direto, mas é veiculado em aula como um ente próximo ao senso comum ou já construído de antemão, sem maiores necessidades de explicações do professor.

Face à falta de um maior aprofundamento envolvendo o conceito de variável nos materiais curriculares direcionados para a Educação Básica, delineamos algumas questões: como poderia ser abordado o conceito de variabilidade para se introduzir o tema das funções? Existiriam outras abordagens do conceito de variável sem necessariamente estar atrelado às funções?
A seguir, passamos a discutir os referenciais que nortearam um esclarecimento face às questões apontadas, de modo a prover um contexto teórico alternativo para se trabalhar o conceito de variável no ensino da Matemática.

\section{$3 \mathrm{O}$ conceito multifacetado de variável}

As pesquisas atuais apontam que o conceito de variável é complexo e possui caráter multifacetado. Matos e Ponte (2008) ponderam que a construção do conceito de variável e a compreensão dos múltiplos sentidos das letras são dois grandes desafios da Educação.

Na Educação Algébrica, passamos a delinear uma contribuição antecipatória aos PCN, Brasil (1997). Estamos nos referindo às referências de Küchemann ${ }^{1}$ (1981). O autor considera seis dimensões para o uso da letra na Matemática: a letra avaliada; a letra não considerada; a letra como objeto; a letra como incógnita; a letra como número generalizado; a letra como variável.

No caso da letra avaliada, esta é substituída por um valor numérico, por tentativa e erro, sem qualquer operação. Por exemplo, ao resolver uma equação $x+4=7$, por tentativa e erro, podese checar que o valor $\mathrm{x}=3$ resolve a equação.

$\mathrm{Na}$ segunda possibilidade, a letra não considerada ocorre quando se reconhece a presença da letra, mas não há a necessidade da respectiva atribuição de significado. No caso da seguinte questão: se $a+b=150$, qual é o valor de $a+b+6$ ? Neste caso, basta reconhecer que a parcela seis (6) deve ser acrescida ao valor 150, obtendo-se 156 como resultado. Deste modo aparecem letras, mas estas não necessitam ser determinadas na resolução.

No caso do uso da letra como objeto, a função é utilizá-la como abreviatura ou como os próprios objetos em si. No caso da expressão ' $3 x^{\prime}$, esta pode ser interpretada como 3 bananas, 3 copos ou 3 objetos, dentre outras possibilidades.

\footnotetext{
Esta interpretação de variáveis como rótulos ou objetos, ao invés de indicadores de quantidades, provou ter uma interpretação muito resiliente. Quando os alunos precisam modelar problemas verbais por meio de equações algébricas, muitas vezes cometem erros com base no uso inapropriado da variável como objeto (GRAY et al., 2005, p. 4).
}

${ }^{1} \mathrm{O}$ autor classificou as dimensões de acordo com os níveis das categorias de Piaget, tomando como base as respostas de alunos em uma pesquisa envolvendo variáveis. 
No quarto do uso da letra como incógnita, muito enfatizada em livros didáticos brasileiros, devem-se reconhecer as letras como números específicos, mas desconhecidos, sobre os quais é possível operar, de acordo com regras préestabelecidas.

Quanto ao uso da letra como número generalizado, cabe se perceber que a letra pode representar vários números, podendo ser substituída por mais do que um valor. Gray et al. (2005) destacam que o uso da letra entendida como número generalizado serve para representar um conjunto finito ou infinito de números, ou ainda estudar as propriedades operatórias da aritmética, como também é um instrumento que permite a observação de regularidades e a expressão de uma generalização de ideias.

No sexto e último caso, a letra como variável corresponde ao ato de notar que a letra pode representar um conjunto de valores (finito ou infinito), cuja mudança provoca uma alteração nas expressões em que está inserida.

Por exemplo, no caso da função de $1^{\circ}$ grau, do tipo $\mathbf{y}=m x+b$, há duas variáveis (' $x^{\prime}$ e ' $y$ '), onde cada letra depende da variação da outra. Em contrapartida, as letras ' $m$ ' e 'b' não são quantidades variáveis, mas representam parâmetros, concebidos como valores específicos, mas que neste tipo de representação permanecem desconhecidos.

Matos e Ponte (2008) destacam que a pesquisa de Küchemann (1981) relata um número considerável de alunos que conseguem um bom desempenho nas questões que exigem o uso da 'letra como incógnita', porém apresentam muitas dificuldades nas categorias 'letra como número generalizado' e 'letra como variável' (p. 198).

Num período posterior, Usiskin (1995) destaca a utilização da letra com distintas finalidades. A primeira concepção é a de Álgebra como Aritmética Generalizada, de modo similar a Küchemann (1981). Um segundo uso se refere à concepção da Álgebra como ferramenta de resolução de problemas, envolvendo a descoberta de um valor particular desconhecido (incógnita) ou de uma constante. Ainda, Usiskin (1995) coloca a Álgebra como estudo de relações entre grandezas: argumento ou parâmetro de uma função. Por último, o autor descreve a Álgebra como estudo das estruturas.
Mais atualmente, Ursini e Trigueros (2001) defendem que as distintas interpretações da linguagem algébrica geram dificuldades aos alunos. Deste modo, estas autoras propõem três categorias principais sobre a sua utilização, $\mathrm{o}$ denominado 'modelo 3UV': incógnita, número generalizado e variável numa relação funcional.

Pode-se perceber que os PCN, Brasil (1998) indicam um modo sincrético do documento oficial brasileiro com relação aos pressupostos delineados nestes três referenciais. Porém, isto representa um grande avanço para o ramo da Educação Algébrica.

Em face destas características, a Educação Algébrica fica favorecida se possibilitarmos aos alunos contextos e situações onde a compreensão do conceito de variável perpassa $o$ reconhecimento e uso flexível da variável nas múltiplas dimensões.

\section{O Referencial Metodológico}

Para viabilizar a pesquisa com variáveis, nos inspiramos na Análise Conteúdo, de Bardin (2004). Deste modo, consideramos a Análise de Conteúdo como o referencial metodológico para a preanálise, coleta e análise de dados.

Para Cury e Konzen (2006), a Análise de Conteúdo é uma metodologia oriunda da área de Comunicação e Ciências Sociais, que estendeu sua atuação ao campo da Educação. O estudo da arte realizado por Ramos e Salvi (2009) indicou que a Análise de Conteúdo está atualmente inserida na área de Educação Matemática, tendo principalmente como sujeitos os discursos de alunos, professores, currículos e livros didáticos.

O objetivo da Análise de Conteúdo é possibilitar a interpretação de mensagens orais ou escritas. Para Beline (2010), na Análise de Conteúdo as mensagens são analisadas sob um referencial dependente do tipo de pesquisa.

Para Bardin (2004), a Análise de Conteúdo se fundamenta na prática, num jogo entre as hipóteses, os objetivos, a aplicação da técnica e a interpretação dos dados. A metodologia se inicia na pré-análise, na qual se escolhem as fontes a serem estudadas: os documentos ou as falas. $\mathrm{Na}$ segunda etapa - a exploração do material - ocorre a desconstrução dos textos escolhidos. Esta fase é um processo de fragmentação, cujo objetivo é perceber os sentidos dos textos, sendo orientada de acordo com os objetivos propostos. 
Na terceira etapa ocorre o tratamento dos resultados e interpretações. Em decorrência, emergem desta fase possíveis unidades de análise, que são resultantes de um processo de interpretação que diversas vezes não se faz em um movimento contínuo.

Para Bardin (2004), estas regras de base apontadas fazem da Análise de Conteúdo um método empírico onde não existe um 'pronto-avestir'. A técnica de Análise de Conteúdo adequada ao objetivo deve ser reinventada a cada momento e se faz em função da área de domínio científico onde será aplicada.

Decidimos nos inspirar nesta metodologia por permitir inferir uma interpretação de um tema - As Equações Diofantinas Lineares - que não está disponível na usual exposição axiomática dos conteúdos de Matemática dos livros didáticos. Na preanálise que realizamos, tomando como pressuposto a necessidade de um novo olhar para o tema, observamos um conjunto de características na exposição usual do tema Equações Diofantinas Lineares nas diversas fontes de conhecimento matemático. Deste modo, neste artigo, temos por hipótese que é possível captar um contexto na exposição das Equações Diofantinas Lineares para elucidar as várias facetas das variáveis, sem necessariamente se iniciar e estar atrelado com o estudo das funções.

Neste sentido, o estudo dos conhecimentos envolvendo as Equações Diofantinas Lineares pode representar um contexto favorável a ser utilizado para delinear as potencialidades do conceito de variável.

\section{A Equação Diofantina Linear e as múltiplas facetas do conceito de variável}

A partir da preanálise dos livros didáticos com relação ao tema das Equações Diofantinas Lineares, destacamos, a seguir, uma das situações encontradas, que, se desenvolvida e explorada, permite apontar os flexíveis usos da variável.

\footnotetext{
Qual sua escolha: CD ou DVD?

Considere a seguinte situação: Michele reserva num certo mês $R \$ 60,00$ para a compra de CDs ou DVDs. Um CD custa R\$ 10,00 e um DVD R\$15,00. Quais são as várias possibilidades de aquisição de Michelle? (STIGLITIZ; WALSH, 2003, p. 27).
}

A importância deste problema se deve principalmente a utilização de um contexto de fácil entendimento e num entorno cotidiano, associado a valores monetários de grandeza numérica adequada, fatores que favorecem e incentivam o aluno para uma busca inicial de algumas ou todas as soluções inteiras, através da manipulação aritmética da tentativa e erro.

A solução deste problema pode se efetivar por simples inspeção numérica, conforme expresso na tabela 01.

Tabela 01- Soluções obtidas

\begin{tabular}{|c|c|c|}
\hline CDs & DVDs & $($ CD;DVD) \\
\hline 0 & 4 & $(0 ; 4)$ \\
\hline 3 & 2 & $(3 ; 2)$ \\
\hline 6 & 0 & $(6 ; 0)$ \\
\hline
\end{tabular}

Ao situar esta perspectiva, há o uso da variável no contexto funcional, no registro pictórico, pois há a possibilidade de verificar que a letra pode representar um conjunto de valores (quantidades de CDs e DVDs), onde a alteração na quantidade de CDs provoca uma alteração nas quantidades de DVDs (figura 01).

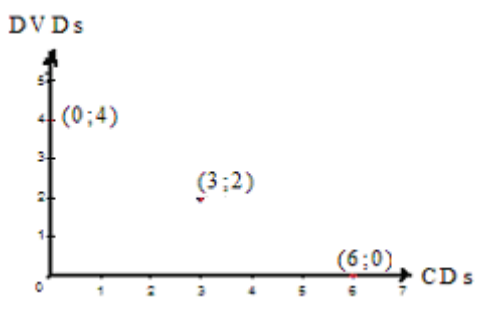

Figura 01: Soluções expressas no Plano Cartesiano

Vale ressaltar outra observação à mobilização das letras no contexto funcional. Na situação esboçada, a forma de utilização da escrita algébrica indica um modo de relacionar grandezas, no viés de letra como variável no contexto funcional. Para Usiskin (1995), a variável se constitui como um argumento, isto é, representa os valores do domínio de certo conjunto, em relação aos valores do contradomínio de outro conjunto.

Nesse sentido, a situação permite explorar o significado da série de valores inteiros da variável 
' $C D$ ' do conjunto $\mathrm{D}=\{0,3,6\}$. Este conjunto se constitui a própria essência do conceito de variável: “Uma letra ' $x$ ' se diz uma variável quando está a individuar todos os elementos de um dado conjunto de números" (MAURER, 1967, p. 7). Do mesmo modo a variável 'DVD' representa os diversos valores presentes no conjunto imagem dado por $\operatorname{Im}=\{0,2,4\}$.

A situação pode ainda ser explorada ao ser explicitada a equação diofantina linear correspondente, dada por $10 x+15 y=60$ " $[\ldots]$ onde $x$ e $y$ representam respectivamente as quantidades de CDs e DVDs possíveis que Michelle poderá adquirir" (STIGLITIZ; WALSH, 2003, p.27).

Esta alternativa de registro algébrico se enquadra no uso da letra como número generalizado, pois pode se perceber que a letra pode representar vários números, podendo ser substituída por mais do que um valor. Ainda, o uso da letra entendida como número generalizado serviu para representar os dois conjuntos finitos - o domínio e a imagem - como também é um instrumento que permite a observação de regularidades: os valores de CDs representam alguns múltiplos naturais de 3 e as quantidades de DVDs indicam uma sequência finita de alguns números naturais múltiplos de 2 .

Vale destacar que, ao se elaborar a representação cartesiana das soluções deste problema, os autores teceram um comentário cuja linguagem aproxima ideias matemáticas importantes, como o caráter discreto das incógnitas envolvidas no problema da restrição orçamentária de Michelle.

\begin{abstract}
Às vezes, obviamente, nem todos os pontos do gráfico são significativos do ponto de vista econômico. É impossível comprar meio DVD ou meio CD. Na maioria dos casos ignoramos essas considerações ao plotar gráficos; consideramos que qualquer ponto da restrição orçamentária é possível (STIGLITIZ; WALSH, 2003, p.36).
\end{abstract}

Essa citação subentende que o traçado da reta tem caráter apenas ilustrativo e de comodidade na representação gráfica da função discreta $\mathrm{f}: \mathrm{N} \rightarrow \mathrm{N}$, que relaciona a variável discreta ' $\mathrm{x}$ ' a variável discreta $y=\frac{12-2 x}{3}$. Este tipo de análise de situação permite ao aluno o entendimento de questões ligadas às quantidades discretas.

De volta à situação discreta dada pelo problema de Michele, a observação do gráfico indica a representação de uma função de $1^{\circ}$ grau. Pode-se aproveitar esta oportunidade e explorar a escrita algébrica na forma $y=m x+n$. No caso, $\mathrm{n}=4$ indica o coeficiente linear, por simples inspeção gráfica. Por outro lado, o valor de ' $\mathrm{m}$ ' indica a tangente do angulo $A$ : $m=\operatorname{tg} \hat{A}=\frac{\Delta y}{\Delta x}=-\frac{4}{6}=-\frac{2}{3}$. Os valores de ' $m$ ' e ' $\mathrm{n}$ ' representam os parâmetros da situação, contrapondo outra forma de uso das letras, agora como parâmetro.

É importante também frisar que a situação problematizada possibilita trabalhar a ideia de coeficiente angular como tangente do ângulo que a reta faz com o eixo Ox sem necessariamente partir do tema funções, mas eventualmente se chegar a ela.

Vale acrescentar que o ensino usualmente coloca como incógnita (ou valor desconhecido) o possível valor de uma equação, que graficamente pode ser visualizado como a raiz da função de $1^{\circ}$ grau: o ponto $(6 ; 0)$.

Esta situação pode ser estendida: nem sempre um consumidor deseja gastar exatamente a quantia máxima disponível. Este tipo de consideração representa o Conjunto de Oportunidades, que significa a lista de todas as opções possíveis ao consumidor para a aquisição de um produto 'A' ou um produto ' $B$ '. A figura 02 representa o caso de Michelle, com gasto de 'até' $\mathrm{R} \$$ 60,00:

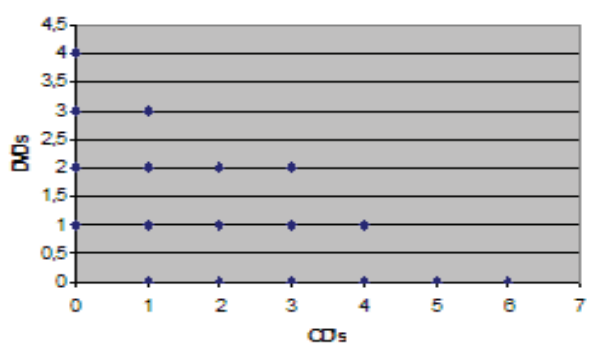

Figura 02: Representação do Conjunto de Oportunidades de Michelle, na forma gráfica.

O Conjunto de Oportunidades é dado pelas soluções da inequação $10 x+15 y \leq 60$, no âmbito dos números inteiros, representado pelos 18 pares ordenados (figura 02). Esta é uma boa oportunidade de abordar a resolução de uma inequação, de modo gráfico, representando soluções inteiras no Ensino Médio e embasadas num contexto cotidiano.

A Restrição Orçamentária representa os limites do Conjunto de Oportunidades, significando os 
pontos mais atraentes no ponto de vista do consumo, visto que possibilita a aquisição de maior quantidade de produtos, dentro do limite de orçamento disponibilizado.

Podemos propor outros valores de gasto, que pode gerar uma solução vazia. Por exemplo, se alterarmos o gasto da aquisição para $\mathrm{R} \$ 70,00$ e os valores unitário de CD e DVD para $R$ \$12,00 e R\$ 16,00, respectivamente, a Equação Diofantina Linear, dada por $12 x+16 y=70$ não apresenta solução.

Tal fato pode ser observado a partir da escrita algébrica $12 x+16 y=70$, que simplificada resulta: $6 x+8 y=35$. Nesta última equação, o primeiro membro resultará num número par, diferindo em paridade ${ }^{2}$ com o segundo membro. Este uso da letra como símbolo abstrato, permite a obtenção de expressões equivalentes e uso/dedução/discussão envolvendo propriedades operatórias da Aritmética e da Álgebra.

\section{Conclusões}

Inicialmente, relembramos que os PCN destacam que:

\begin{abstract}
Aprender Matemática no Ensino Médio deve ser mais do que memorizar resultados dessa ciência e que a aquisição do conhecimento matemático deve estar vinculada ao domínio de um saber fazer Matemática e de um saber pensar matemático (BRASIL, 1998, p. 41).
\end{abstract}

No desenvolvimento progressivo do aluno ao longo da sua Educação Algébrica, o papel desempenhado pela linguagem envolvendo os símbolos:

[...] desempenham um papel fundamental na constituição do pensamento algébrico abstrato, uma vez que ela fornece um simbolismo conciso por meio do qual é possível abreviar o plano de resolução de uma situação-problema, o que possibilita dar conta da totalidade e da estrutura da organização (BRASIL, 1998, p. 89).

O conceito de variável representa um importante momento a se desenvolver o pensamento algébrico. Ameron (2002) aponta que exploração do uso flexível da letra na Álgebra estimula aspectos essenciais do pensamento algébrico, pois representa uma possibilidade de estabelecer relações envoltas em um processo que envolve o uso de incógnitas,

\footnotetext{
${ }^{2}$ Dois números inteiros têm mesma paridade, quando são
} ambos pares ou ambos ímpares. relações generalizadoras e abstratas, entre grandezas, no viés funcional.

A autora argumenta que o pensamento algébrico associado à simbolização algébrica permite que o estudante adquira um entendimento mais amplo da Álgebra e, em particular, do conceito de variável.

Historicamente, o conceito de variável emergiu a partir da simbolização da Álgebra.

\begin{abstract}
Diofanto foi pioneiro na resolução de equações indeterminadas. Os matemáticos hindus, sobretudo a partir do século II d.C., desenvolveram uma Álgebra mais simbólica do que a de Diofanto, avançando também na resolução de equações indeterminadas (BOTELHO; REZENDE, 2009, p. 5).
\end{abstract}

A análise do contexto epistemológico envolvendo o tema das Equações Diofantinas Lineares revelou um possível caminho para expressar o uso das letras na Educação Algébrica.

No contexto funcional, a exploração de problemas no contexto das Equações Diofantinas Lineares viabiliza o uso da variável pela expressão das grandezas envolvidas no registro tabular e gráfico, porém ainda restrita a uma concepção estática das variáveis envolvidas.

O registro do domínio e imagem das variáveis permitiu constituir a própria essência do conceito de variável, pois a variável permite individuar todo e qualquer elemento de certo conjunto numérico.

Ao analisarmos a situação proposta, no viés de uso da letra como número generalizado, foi possível perceber um padrão de regularidade nos conjuntos representados pelo domínio e pela imagem.

Esta visão de letra como número generalizado, associada ao registro gráfico no viés funcional, favorece uma concepção dinâmica para a variável. No contexto da situação envolvendo o tema da Equação Diofantina Linear isto é determinado pela mudança que certo valor da variável ' $x$ ' pode causar nos valores de ' $y$ ', porém estas relações estão regidas e reguladas pela lei de expressão algébrica.

A própria expressão algébrica pode ser reapresentada na forma funcional, pela possibilidade de explicitação do coeficiente linear e angular, que favoreceram um quadro de mudança de registro da equação diofantina linear para a expressão de função de $1^{\circ}$ grau. 
Ainda, a extrapolação do tema das Equações Diofantinas Lineares permitiu situar um quadro de inequações de $1^{\underline{0}}$ grau em um contexto de grandezas discretas. Nessa situação, o Conjunto de Oportunidades viabilizou uma exposição, pouco usual no ensino básico, enriquecendo o repertório do uso das letras, num viés abstrato.

Outra forma de desenvolver o viés abstrato foi viabilizado pela mudança de valores dos parâmetros, que estabeleceu um quadro da inexistência de solução. Este aspecto implicou na exploração de propriedades mais gerais, que estimula o uso das letras como símbolo abstrato.

Pudemos constatar que a análise de conteúdo proposta por Bardin (2004) possui uma função heurística. Nesse sentido, o estudo do tema das Equações Diofantinas Lineares representou um movimento de abertura a novas formas de abordagem no campo da didática, na área da educação matemática.

Em outra posição, a análise de conteúdo possui uma função de administração da prova. Nesse sentido, esta metodologia proveu uma diretriz para testar certas hipóteses, aliando o rigor da pesquisa à necessidade de ir além das aparências, permitindo a exploração da polaridade rigor e exploração. Deste modo, a interpretação do viés múltiplo do conceito de variável pela exposição das características essenciais das Equações Diofantinas Lineares serviu como uma espécie de prova, delineada pela análise situada no caminho de uma possível confirmação ou refutação.

No presente caso, as Equações Diofantinas Lineares delimitaram aspectos propícios para se explorar o uso das letras na Álgebra de modo mais flexível e conceitual, tecendo uma rede de relações de significação entre os múltiplos usos do conceito multifaceta de variável.

Acreditamos que a exploração das Equações Diofantinas Lineares negocia uma abertura para uma busca de outros temas, dentro da própria área da Matemática, que poderiam favorecer a Educação Algébrica.

As variações obtidas pela mudança de valores em situações problematizadas permitem delinear momentos em que o uso das letras em determinado viés - incógnita; aritmética generalizada, funcional e abstrata - podem ser mais favoráveis e permitam significar os símbolos perante os próprios conhecimentos matemáticos.

\section{Referências}

AMERON, B. A. van (2002). Reinvention of Early Algebra: Developmental research on the transition from arithmetic to algebra. Utrecht: Freudenthal Institut.

BARDIN, L. (2004). Análise de conteúdo. 3. ed. Lisboa Edições.

BELINE, W. (2010). ANÁLISE DE CONTEÚDO E OS SENTIDOS DO PROCEDIMENTO "VAI UM" NA OPERAÇÃO DE ADIÇÃO PARA FORMANDAS EM PEDAGOGIA. V EPCT.

BOTELHO, L., REZENDE, W. (2011). Um Breve Histórico do Conceito de Função. Caderno DáLicença, pp. 64-75.

BRASIL. (1997). Parâmetros Curriculares Nacionais: Matemática. Brasília: SEMT/MEC.

(1998). Parâmetros Curriculares Nacionais: $3^{\text {o }}$ e $4^{\circ}$ ciclos do Ensino Fundamental. Brasília: SEMT/MEC.

(2000). Parâmetros Curriculares Nacionais para o Ensino Médio. Brasília: SEMT/MEC.

(2005). PCN+: Orientações Educacionais Complementares aos Parâmetros Curriculares Nacionais. Brasília: SEMT/MEC.

CAMPBELL, S., ZAZKIS, R. (2002). Toward Number Theory as a Conceptual Field. In: CAMPBELL, S.; ZAZKIS, R. (org.). Learning and Teaching Number Theory. London: Ablex Publishing. Cap. 1. pp. 1-14.

CASTRO, M. R. (2005). Educação Algébrica e Resolução de Problemas. Disponível em: $<$ http://www. tvebrasil.com.br/salto $>$. Acesso em: 01 out. 2011.

CHEVALLARD, Y., BOSCH, M., GASCÓN, J. (2001). Estudar Matemáticas: O elo perdido entre o ensino e a aprendizagem. Tradução de: Daisy Vaz de Moraes. Porto Alegre: ArtMed.

COURANT, R., ROBBINS, H. (2000). O que é Matemática? Tradução de: Adalberto da Silva Brito. Rio de Janeiro: Ciência Moderna.

GRAY, S. S., LOUD, J. L., SOKOLOWSKI, C. P. (2007). Calculus Students' Difficulties in Using Variables as Changing Quantities. Disponível em:

$<$ http://sigmaa.maa.org/rume/crume2007/papers/ 
gray-loud-sokolowski.pdf>. Acesso em 12 mai. 2014.

KÜCHEMANN, D. (1981). Algebra. In: HART, K. (ed.). Children's understanding of mathematics. London: John Murray.

MATOS, A., PONTE, J. P. (2008). O estudo de relações funcionais e o desenvolvimento do conceito de variável em alunos do $8^{\mathrm{o}}$ ano. Relime, México, v. 11, n. 2, pp. 195-231. Disponível em: $<$ http://www.scielo.org.mx/scielo.php?script=sci arttext\&pid=S166524362008000200003\&lng=es\&n $\underline{\mathrm{rm}=\mathrm{iso}>}$. Acesso em 14 mai. 2014.

MAURER, W. A. (1967). Curso de Cálculo Diferencial e Integral. São Paulo: Edgard Blucher, v.1.

RAMOS, R. C. S. S., SALVI, R. F. (2009). Análise de Conteúdo e Análise do Discurso em Educação Matemática: Um olhar sobre a produção em periódicos Qualis A1 e A2. VI SIPEM.

SÃO PAULO. (2008). Proposta Curricular do Estado de São Paulo: Matemática. São Paulo: SEE.

SCHOENFELD, A. H., ARCAVI, A. (1998). On the meaning of variable. Mathematics Teacher, pp. 420-427.

SILVA, L. M., ANDRADE, S. (2012). A Construção do Conceito de Função e o Contrato Didático.

STIGLITIZ, J. E., WALSH, C. E. (2003). Introdução à Microeconomia. 3. ed. Rio de Janeiro: Editora Campus.Tradução de: Priciples of Microeconomics, 3rd ed.

URSINI, S., TRIGUEIROS, M. (2001). A Model for the uses of variable in elementary algebra. In: M. Van den Heuvel-Panhuizen (ed.), Proceedings of the $25^{\text {th }}$ International Conference for the Psychology of Mathematics Education. Utrecht, Netherlands, v. 4.

USISKIN, Z. (1995). Concepções sobre a Álgebra da escola média e utilizações das variáveis. In: COXFORD, A. F., SHULTE, A. P. (org). As Ideias da Álgebra. São Paulo: Atual. 